\title{
REVIEW
}

\section{DNA fragmentation in apoptosis}

\author{
Zhang Jian Hua, Ming XU* \\ Department of Cell Biology, Neurobiology and Anatomy, Uni- \\ versity of Cincinnati College of Medicine, Cincinnati, Ohio \\ 45267-0521, USA
}

\begin{abstract}
Cleavage of chromosomal DNA into oligonucleosomal size fragments is an integral part of apoptosis. Elegant biochemical work identified the DNA fragmentation factor (DFF) as a major apoptotic endonuclease for DNA fragmentation in vitro. Genetic studies in mice support the importance of DFF in DNA fragmentation and possibly in apoptosis in vivo. Recent work also suggests the existence of additional endonucleases for DNA degradation. Understanding the roles of individual endonucleases in apoptosis, and how they might coordinate to degrade DNA in different tissues during normal development and homeostasis, as well as in various diseased states, will be a major research focus in the near future.
\end{abstract}

Key words: DNA fragmentation, $D F F$, endonucleases,

apoptosis.

Apoptosis, or programmed cell death, has been used to describe a form of cell death in an active and inherently controlled manner that eliminates no longer wanted cells [1]. Cell and nuclear shrinkage, chromatin condensation, formation of apoptotic bodies and phagocytosis by neighboring cells characterize the main morphological changes of the apoptosis process[1]. Cleavage of chromosomal DNA into oligonucleosomal size fragments is a biochemical hallmark of apoptosis[2]. The extensive morphological and

\footnotetext{
* Correspondence to: Ming Xu, Ph.D.

Tel: 513-558-2922; Fax: 513-558-4454; E-mail: ming.xu@uc.edu
} 
DNA fragmentation in apoptosis

biochemical changes during apoptosis are likely to ensure that dying cells would leave minimum impact to neighboring tissues in vivo. These features of apoptosis contrast those of necrosis, another form of cell death in response to noxious stimuli or injury. Necrosis is accompanied by membrane rupture and leakage of cellular contents, and it thus often causes tissue inflammation. During normal development and tissue homeostasis, majority of the cells are believed to die through apoptosis. Moreover, dysregulation of apoptosis seems to be directly linked to human diseases including cancer, autoimmune diseases, and neuronal degeneration[3-5]. Therefore, understanding the mechanisms of apoptosis is not only driven by an intellectual curiosity about a fascinating fundamental cellular process, but also by urgent needs to find treatment strategies for the dreaded diseases.

Shortly after the initial description of apoptosis, a series of elegant genetic analyses in C. elegans revealed two genes, ced- 3 and ced- 4 , that are required for programmed cell death during worm development[6]. Subsequent studies uncovered a cellular suicide program and additional molecular components of the apoptotic machinery. For example, the product for ced- 3 is a caspase, whose activation is promoted by the ced- 4 product, whereas ced-9 functions upstream to negatively regulate apoptosis. The cell death program is highly conserved from C. elegans to humans, and mammalian counterparts of ced- 3, ced- 4 and ced- 9 have all been found as caspases, Apaf-1 and Bcl-2 family of genes, respectively. Further work demonstrated that the molecular events underlying mammalian programmed cell death include regulation of apoptotic signals by Bcl-2 family of proteins and initiator caspases, cytochrome c release, downstream caspase activation, chromatin condensation, DNA fragmentation and phagocytosis of dead cells by scavenger cells[7]. Despite this knowledge, the identity of the molecular machinery for DNA fragmentation and consequently the role of DNA fragmentation in apoptosis remained elusive.

\section{The DNA fragmentation factor degrades chromosomal DNA in vitro}

In an effort to identify the apoptotic endonuclease(s), Wang and colleagues used a clever in vitro biochemical assay system and purified a protein that they termed the DNA fragmentation factor, or DFF[8]. DFF is a heterodimeric protein composed of DNA fragmentation factors 45 and 40 , and it is capable of causing chromosomal DNA fragmentation in the presence of an activated caspase 3 (Fig 1). Very shortly afterwards, three groups showed further that DFF40 contains an intrinsic DNase activity whereas DFF45 serves as an inhibitor of DFF40 activity[9-14]. Upon activation of apoptosis, DFF45 is cleaved by caspase 3, and dissociates from DFF40[8-14]. DFF40 or the caspaseactivated DNase or nuclease (CAD or CPAN) is now activated and is capable of cleaving DNA into oligonucleosomal size fragments (Fig 1). Subsequent work showed that DFF45 could also be activated by caspase 7 and granzyme B[12],[15]. Activated DFF40 can generate double stranded breaks and its activity can be potentiated by high-mobility 
group proteins, as well as by histone H1[12]. DFF45 is required for the synthesis and folding of DFF40, thus serving also as a molecular chaperone[8,10, 14]. Both DFF40 and DFF45 are found in human, mouse and Drosophila, while no homologs have been reported in C. elegans[14]. These biochemical studies indicate that all the properties associated with DFF fit those of an apoptotic endonuclease nicely.
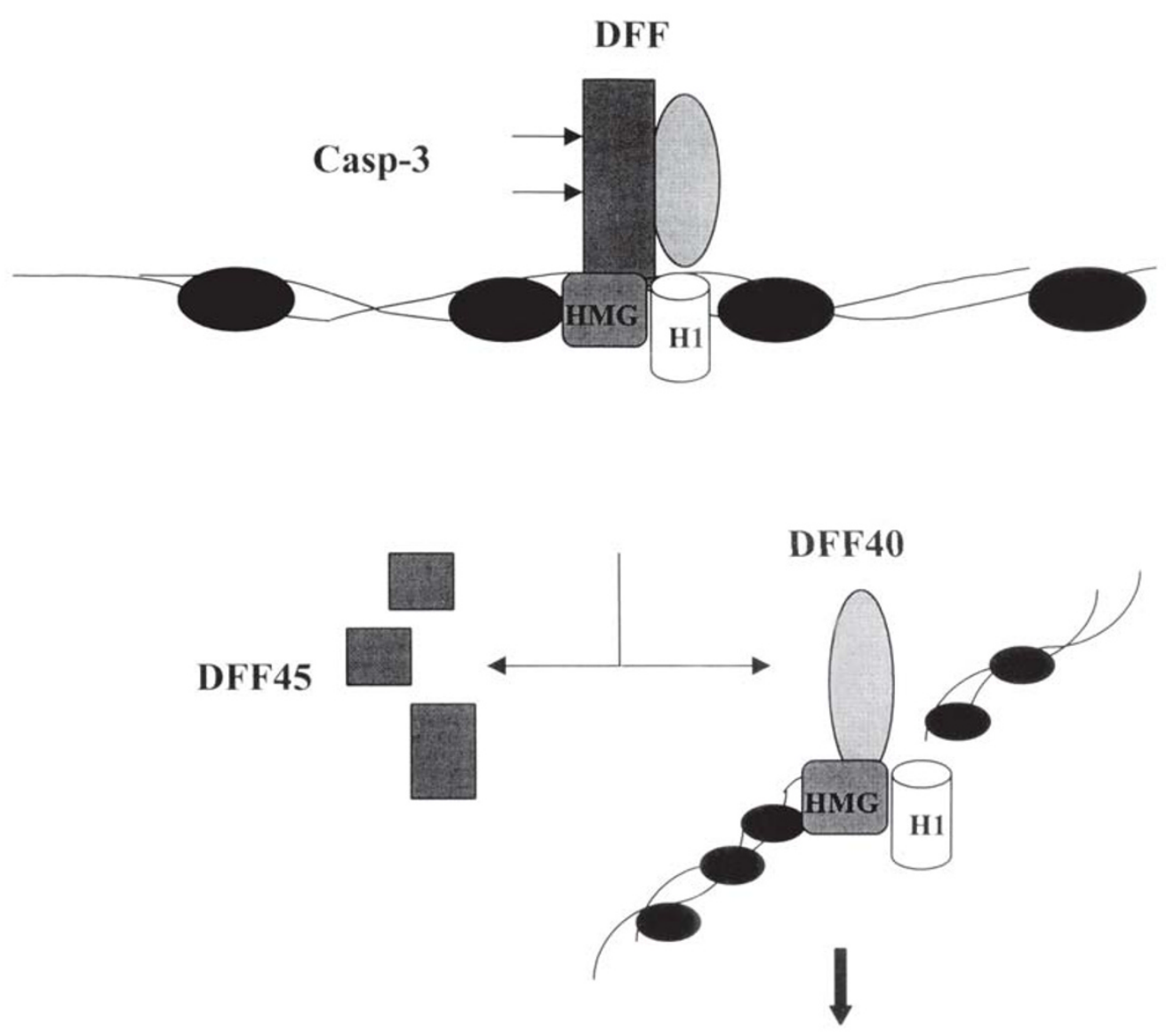

DNA fragmentation

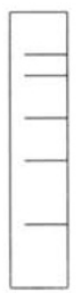

Fig 1. DNA fragmentation during apoptosis. DFF is a heterodimeric protein composed of DNA fragmentation factors 45 and 40. Upon activation of apoptosis, DFF45 is cleaved by caspase 3 and dissociates from DFF40. The DFF40 endonuclease is activated and cleaves chromosomal DNA at internucleosomal sites. High-mobility group (HMG) proteins and histone H1 can potentiate DFF40 endonuclease activity. 
DNA fragmentation in apoptosis

\section{The DNA fragmentation factor serves as a major apoptotic endonuclease in vivo}

To investigate the in vivo role of DFF45 in DNA fragmentation and apoptosis, we used gene targeting and generated DFF45 mutant mice[16]. Extracts from various tissues of the DFF45 mutant mice were devoid of DNA fragmentation activities when added to rat liver nuclei in the presence of an activated caspase 3 in an in vitro assay system[16]. This result indicates that DNA fragmentation activity is lost due to the DFF45 mutation. Further analysis demonstrated that, in response to various apoptotic stimuli, different types of DFF45 deficient cells isolated from the mutant mice were resistant to DNA fragmentation[16]. Moreover, dexamethasone injections also failed to induce DNA fragmentation in DFF45 deficient thymus in vivo[17]. These experiments, together with the previous biochemical experiments, clearly established that DFF plays a crucial role in DNA fragmentation in various cells upon activation of apoptosis in vivo. Further supporting this conclusion is the result that DNA fragmentation was abolished in cells or transgenic mice carrying an uncleavable form of DFF45[18],[19].

In addition to DNA degradation, DFF has also been shown to induce chromatin condensation in vitro[10]. Our work using DFF45 deficient cells supports this claim in that there appeared to be less chromatin condensation in the mutant cells than in normal control cells upon activation of apoptosis[16]. Furthermore, electron microscopic studies demonstrated that dying DFF45 deficient cells exhibit different morphology than normal control cells in that they show reduced degree of chromatin condensation, absence of nuclear fragmentation, intranuclear cytoplasmic invagination and striking nuclear chromatin conglutination after release from disintegrating cells[17]. These results argue that in addition to DNA fragmentation, DFF also participates in other events in apoptosis.

\section{How important is it to degrade chromosomal DNA in vivo?}

DFF40 and 45 are expressed ubiquitously in mouse tissues throughout development, suggesting it may also function during this time period[17]. Nevertheless, it has been shown that cell nucleus and DNA fragmentation are not required for apoptosis under certain conditions[20]. We also observed that apoptosis in DFF45 deficient cells could occur despite the resistance to DNA fragmentation and chromatin condensation[16], [17]. Why then an endonuclease system(s) evolved to degrade chromosomal DNA during apoptosis? To address this issue, we investigated the possible in vivo consequences of decreased DNA fragmentation in all major organ systems during mouse development by performing a systematic histological survey using DFF45 mutant mice. There were no significant alterations including hypertrophy in DFF45 deficient organs when compared to those from normal control mice[16]. A quantitative analysis of the peripheral blood cells and immune cells also revealed that the presence of DFF45 is not obligatory 
for normal hematopoeisis and immune system deelopment[16].

When we compared the viability of cells from DFF45 mutant and normal control mice upon activation of apoptosis, we found that DFF45 deficient cells exhibit a slower rate of apoptosis than that of the normal control cells in response to multiple apoptotic stimuli[17]. This result suggests that the cleaved DFF45 and/or the double stranded DNA breaks during DNA fragmentation can promote efficient apoptosis[17]. To examine whether the apparent resistance to apoptosis in DFF45 deficient cells may correlate with any effects in the central nervous system where timely apoptosis is crucial during development, we counted and compared neuronal numbers in the hippocampus of DFF45 mutant and normal control mice. We found that both the granule cell density and total granule cell number in the hippocampal dentate gyrus region are higher in the DFF45 mutant brains than in the normal control brains[21]. The increased granule cell number correlated with enhanced spatial learning and memory in DFF45 mutant mice compared to control mice[21]. Although the direct cause of the increased neuronal number and the enhanced spatial learning is still unclear, it may well be that in the absence of DFF45, a change in timely neuronal cell death affected proper wiring of the central nervous system, resulting in a change in neuronal function. These studies demonstrate that DFF45 is clearly important for DNA fragmentation, and in at least some cases, timely cell death or even cellular function. The fact that DFF45 mutant mice are generally devoid of dramatic abnormalities also suggests either that a lack of DNA fragmentation and cell death may not be detrimental to development and tissue homeostasis, or that other endonucleases may exist to degrade DNA during apoptosis in these mice.

\section{Are there other endonucleases for DNA degradation?}

Two recent findings provided new clues to answer this question. Horvitz and colleagues showed that a complete DNA degradation requires activities both from engulfing cells and from NUC-1 in dying cells[22]. NUC-1 is a homolog of DNase II and it plays an important role in an intermediate step of DNA degradation upon activation of apoptosis in C. elegans[22]. At the same time, Nagata and colleagues reported that, in a dominant-negative DFF45 transgenic mouse in which the DFF activity is inhibited, DNA fragmentation could occur in phagocytes[19]. Moreover, DNase II residing in the lysosomes of the phagocytes is the likely endonuclease for degrading DNA after the apoptotic cells have been engulfed[19]. It should be noted that the cellular location of DNase II makes it difficult to be a major player in cell autonomous apoptosis at least in mammalian systems. For the same reason, DNase II could be equally involved in both apoptosis and necrosis. Nevertheless, these two new findings point to the possibility that, in addition to DFF, there are backup systems to degrade chromosomal DNA in dying cells during apoptosis.

Additional candidate endonucleases have also been proposed to degrade DNA during apoptosis. DNase I presumably could degrade any DNA that are extracellular[23]. Cyclophilins can digest nuclear DNA[24]. The inducible-lymphocyte $\mathrm{Ca}^{2+} / \mathrm{Mg}^{2+}$-depen- 
DNA fragmentation in apoptosis

dent endonuclease is up-regulated during $\mathrm{T}$ cell apoptosis[25]. A key aspect with all these candidate endonucleases is that their in vivo involvement in apoptosis will need to be firmly established. In addition, yet unidentified endonucleases may exist which could contribute to chromosomal DNA degradation during apoptosis.

\section{Unresolved future issues}

The fact that multiple ways may exist to ensure a complete DNA degradation during apoptosis suggests that DNA fragmentation is a crucial biological event. Uncovering the significance of each of these systems in DNA degradation during apoptosis will continue to be a major research focus. Moreover, when apoptosis is in overwhelming demands, such as after brain ischemia or traumatic injuries, after chemical carcinogen or irradiation treatment, or after massive viral or bacterial infections, prompt DNA fragmentation can become of paramount importance in ensuring timely apoptosis, in removing potentially harmful DNA and in maintaining genome integrity. Furthermore, in the presence of oncogenic mutations when the tissues are prone to tumorigenesis, a lack of proper DNA fragmentation could exacerbate the process. The DFF45 mutant mice could provide a useful system to address these issues. In the near future, we should have a much clearer picture of the roles of individual endonucleases in apoptosis, and how they might coordinate to degrade DNA in different tissues during normal development and homeostasis, as well as in various diseased states. This knowledge may provide new treatment strategies to prevent or treat human diseases.

\section{ACKNOWLEDGMENTS}

We sincerely thank Xiaodong WANG for continuous advice and stimulating discussions. J.Z. is an Effie D. Beeman National Alliance for Research on Schizophrenia and Depression investigator and is supported by NIDA (DA11284). M. X. is a National Alliance for Research on Schizophrenia and Depression investigator and is supported by NIDA (DA11005).

\section{REFERENCES}

[1] Kerr JFR, Wyllie AH, Currie AR. Apoptosis: a basic biological phenomenon with wide-ranging implication in tissue kinetics. Br J Cancer 1972; 26:239-57.

[2] Wyllie AH. Glucocorticoid induced thymocyte apoptosis is associated with endogenous endonuclease activation. Nature 1980; 284:555-6.

[3] Thompson CB. Apoptosis in the pathogenesis and treatment of disease. Science 1995; 267:1456-62.

[4] Vaux DL, Korsmeyer SJ. Cell death in development. Cell 1999; 96:245-54.

[5] Nijhawan D, Honarpour N, Wang X. Apoptosis in neural development and disease. Annu Rev Neurosci 2000; 23:73-87.

[6] Ellis RE, Yuan JY, Horvitz HR. Mechanisms and functions of cell death. Annu Rev Cell Biol 1991; 7: 663-98.

[7] Budihardjo I, Oliver H, Lutter M, Luo X, Wang X. Biochemical pathways of caspase activation during 
apoptosis. Annu Rev Cell Dev Biol 1999; 15:269-90.

[8] Liu X, Zou H, Slaughter C, Wang X. DFF, a heterodimeric protein that functions downstream of caspase3 to trigger DNA fragmentation during apoptosis. Cell 1997; 89:175-84.

[9] Enari M, Sakahira H, Yokoyama H, Okawa K, Iwamatsu A, Nagata S. A caspase-activated DNase that degrades DNA during apoptosis, and its inhibitor ICAD. Nature 1998; 391:43-50.

[10] Liu X, Li P, Widlak P et al. DFF40 induces DNA fragmentation and chromatin condensation during apoptosis. Proc Natl Acad Sci USA 1998; 95:8461-6.

[11] Sakahira H, Enari M, Nagata S. Cleavage of CAD inhibitor in CAD activation and DNA degradation during apoptosis. Nature 1998; 391:96-9.

[12] Liu X, Zou H, Widlak P, Garrard W, Wang X. Activation of the apoptotic endonuclease DFF40 (caspaseactivated DNase or nuclease). J Biol Chem 1999; 74:13836-40.

[13] Halenbeck R, MacDonald H, Roulston A, Chen TT, Conroy L, Williams LT. CPAN, a human nuclease regulated by the caspase-sensitive inhibitor DFF45. Curr Biol 1998; 8:537-40.

[14] Nagata S. Apoptotic DNA fragmentation. Exp Cell Res 2000; 256:12-8.

[15] Thomas D, Du C, Xu M, Wang X, Ley TJ. DFF45/ICAD can be directly process by granzyme B during the induction of apoptosis. Immunity $2000 ; \mathbf{1 2}: 621-32$.

[16] Zhang J, Liu X, Scherer DC, Van Kaer L, Wang X, Xu M. Resistance to DNA fragmentation and chromatin condensation in mice lacking the DNA fragmentation factor 45. Proc Natl Acad Sci USA 1998; 95:12480-5.

[17] Zhang J, Wang X, Bove KE, Xu M. DNA fragmentation factor 45-deficient cells are more resistant to apoptosis and exhibit different dying morphology than wild- type control cells. J Biol Chem 1999; 274: 37450-4.

[18] Sakahira H, Enari M, Ohsawa Y, Uchiyama Y, Nagata S. Apoptotic nuclear morphological change without DNA fragmentation. Current Biology 1999; 9:543-6.

[19] McIlroy D, Tanaka M, Sakahira H et al. An auxiliary mode of apoptotic DNA fragmentation provided by phagocytes. Genes Dev 2000; 14:549-58.

[20] Schulze-Osthoff K, Walczak H, Droge W, Krammer PH. Cell nucleus and DNA fragmentation are not required for apoptosis. J Cell Biol 1994; 127:15-20

[21] Slane J, Lee H, Vorhees C, Zhang J, Xu M. DNA fragmentation factor 45 deficient mice exhibit enhanced spatial learning and memory compared to wild-type control mice. Brain Res 2000; 867:70-9.

[22] Wu Y-C, Stanfield GM, Horvitz HR. NUC-1, a Caenorhabditis elegans Dnase II homolog, functions in an intermediate step of DNA degradation during apoptosis. Genes Dev 2000; 14:536-48.

[23] Montague JW, Hughes Jr FM, Cidlowski JA. Native recombinant cyclophilins A, B, and C degrade DNA independently of peptidylprolyl cis-trans-isomerase activity. J Biol Chem 1997; 272:6677-84.

[24] Peitsch MC, Polzar B, Stephan H et al. Characterization of the endogenous deoxyribonuclease involved in nuclear DNA degradation during apoptosis (programmed cell death). EMBO J 1993; 12:371-7.

[25] Khodarev NN, Ashwell JD. An inducible lymphocyte nuclear $\left.\mathrm{Ca}^{2+} / \mathrm{Mg}^{(2+}\right)$-dependent endonuclease associated with apoptosis. J Immunol 1996; 156:922-31.

Received June-27-2000. Revised July-6-2000. Accepted July-17-2000. 